\title{
Performance and stability of a field fibre optics current sensor
}

\author{
F. Briffod, L. Thévenaz and Ph. Robert \\ EPFL Swiss Federal Institute of Technology, Metrology Laboratory, $\mathrm{CH}-1015$ Lausanne, Switzerland \\ Tel : [++41 21] 6937606 Fax : [++41 21] 6932614 E-mail : fabien.briffod@epfl.ch
}

Abstract : Performance and stability of a field fibre optics interferometric current sensor are presented, showing a $\pm 0.5 \%$ accuracy over a broad temperature range and an excellent insensitivity to the sensing fibre positioning.

\section{INTRODUCTION}

This paper presents the latest evaluations performed on an interferometric Sagnac fibre optics current sensor developed by the Metrology Laboratory in the Swiss Federal Institute of Technology, in collaboration with Trench Switzerland AG. This sensor is designed to measure high DC and AC currents up to $\pm 500 \mathrm{kA}$. The objective of this sensor is to obtain a $\pm 0.5 \%$ accuracy over the full $\pm 500 \mathrm{kA}$ range, for a temperature range between 0 and $50 \operatorname{degC~(-20~to~} 80 \mathrm{degC}$ for the sensing fibre), to comply with the end user requirements.

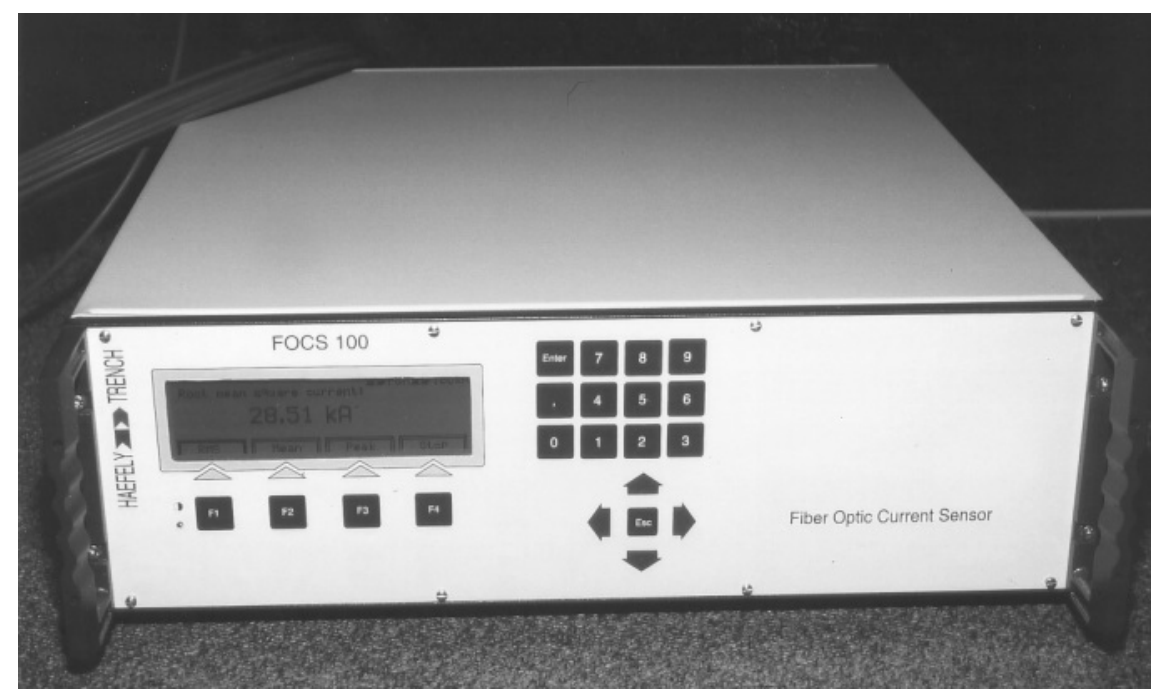

Figure 1: Sagnac fibre optics current sensor ready for field measurements. The sensing cable that must enclose the probed electrical conductor is partially shown on top-left of the figure.

As a result of the developments on optical gyroscopes [1,2] it is now well-known that the Sagnac interferometer has the key advantage to be only sensitive to nonreciprocal effects, such as rotation and Faraday effect. As a result, this interferometer is intrinsically insensitive to reciprocal effects such as fibre elongation due to thermal dilatation or mechanical solicitations.

The main issue that prevented the development of a reliable instrument so far is related to the fibre birefringence. It turns out that such a sensor can only give unbiased measurements, provided that the light polarisation is maintained circular all along the sensing fibre loop. Many solutions have been proposed to compensate the effect of birefringence, either optically or through a set of measurements and some calculations $[3,4,5]$. But these techniques are limited to homogeneous linear birefringence along the sensing fibre, so that they turn out to be widely inapplicable in actual conditions in which the fibre birefringence is basically random.

Fortunately the detrimental effect of polarisation mode dispersion in telecommunication systems had led to a big effort for manufacturing very low birefringence fibres at low cost. The remaining birefringence of such fibres turns out to be still too large for the proper operation of a current sensor. But it can now be widely rendered negligible by annealing [6] or mechanically twisting the fibre [7], so that a circular or freely-rotating linear polarisation is maintained over the entire fibre length. The aim of this paper is in particular to demonstrate that fibre birefringence is a solved problem as far as the fibre current sensor is concerned, so that a flexible instrument can be realised with a simple handling for the end user. 


\section{SETUP}

The diagram of the realised fibre current sensor is shown in Figure 2. The configuration is very similar to that used in fibre optics gyroscopes, with the particularity to propagate the light through the sensing loop using a circular polarisation. This particularity requires a proper preparation of the light polarisation. For current sensing the fibre loop must enclose the probed electrical conductor. The circulation of the current magnetic field along the fibre causes a phase lag between circularly polarised waves propagating in opposite directions, as a result of the Faraday effect. After propagation into the sensor head, the two waves recombine, resulting in an interference signal to be processed.

In absence of any electrical current the interferometer is fully balanced and its sensitivity to a differential phase shift is zero. An electro-optic phase modulator is thus used to bias the response of the interferometer, so that a maximum sensitivity for small electrical current is obtained, technique similar to that used in fibre gyros [8]. A standard lock-in detection at $f_{m}$ and $2 f_{m}$ followed by a suitable signal processing provides a value for the electrical current independent on any change in the light source intensity. The electro-optic phase modulation turns out to be temperature-dependent to an unacceptable extent, so that the Gyro-type modulator has to be temperature-controlled. The electro-optic modulator also achieves the splitting-recombination functions of the interferometer and transmits the light only in a well defined linear polarisation state.

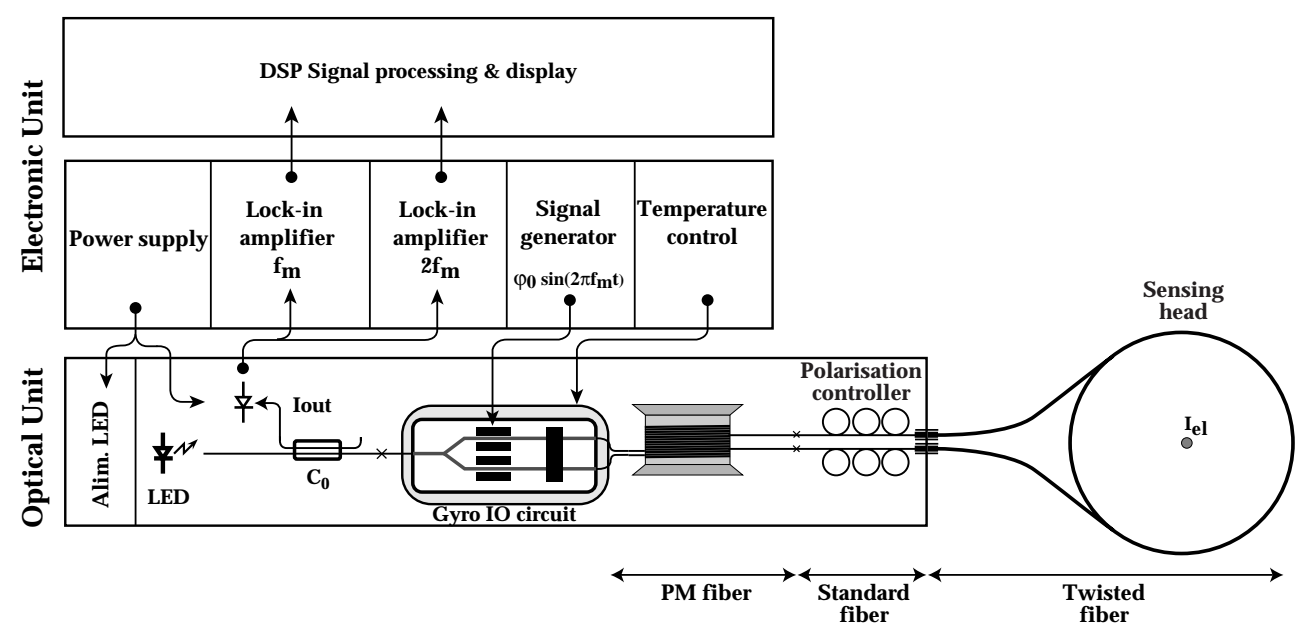

Figure 2: Block diagram of the Sagnac interferometric current sensor in the field measurement configuration.

Polarisation maintaining fibres (PM fibre) are used to transmit the linearly polarised light from the electro-optic phase modulator (GYRO circuit) to high stability polarisation controllers dedicated to the preparation of the proper polarisation for the sensing fibre. With respect to classical configurations some functional improvements were brought up. The sensing fibre is a simple cable containing a mechanically twisted fibre and terminated with connectors. Hence, on-site installation is easily performed by wrapping the fibre around the conductor and does not require the interruption of the probed electrical circuit. To grant a circular polarisation in the sensing head, polarisation controllers are carefully set, so that they transform the linear polarisation into a circular polarisation within the sensing fibre, by moreover compensating any spurious birefringence induced by the connectors.

\section{MEASUREMENTS}

The performances obtained using the field sensor are presented in this section. Figure 3 shows the response of the sensor up to $100 \mathrm{kA}$, obtained for DC current, demonstrating the excellent linearity of the instrument. But thermal and mechanical stability and reproducibility are key-issues for fibre current sensors and a full set of measurement was carried out to emphasise problems related to these issues.

Figure 4 shows the fluctuations of the measured value at turn-on and on a long-term basis, at constant ambient temperature. The measurement change is defined as $100 \cdot\left(I_{\text {mes }}-I_{\text {ref }}\right) / I_{\text {ref }}[\%]$. A $\pm 0.2 \%$ drift is observed, and accurate operation is obtained after a less than 5 minute turn-on transient time. The scattering of the points is not representative of the intrinsic noise of the system, but is an experimental artefact due to the time lag between the reference and the optical measurements. 


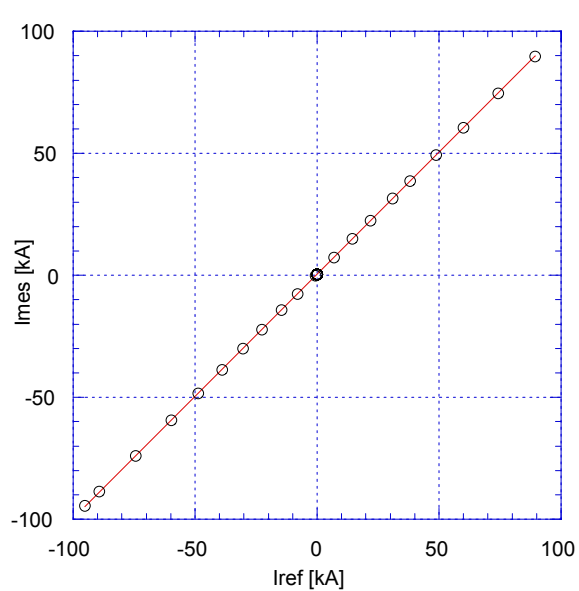

Figure 3: Response of the sensor to DC current up to 100kA. $I_{r e f}$ is the reference electrical current and $I_{m e s}$ is the optically measured current.

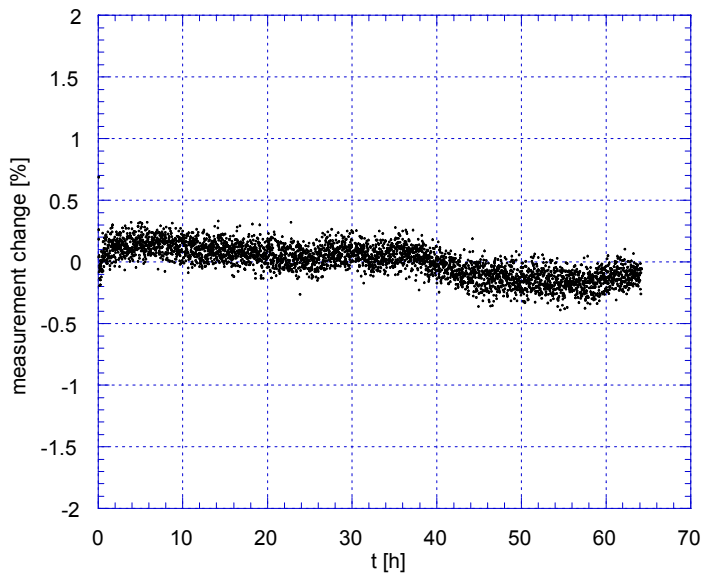

Figure 4: Turn-on transient and long-term measurement. Points Scattering is not representative of system noise, but is due to the delay between reference and optical measurements.

Figure 5 shows the measurement change when the cable is strongly manipulated and its position significantly modified. This critical measurement is a clear indicator of the capability of the sensing fibre to maintain the circular polarisation. In our case the measurements lie just within the $\pm 0.5 \%$ limit, but this figure could be most probably improved by a more important mechanical fibre twisting. Such a change may also indicate that the polarisation states within the fibre are not exactly set to circular as a result of an inaccurate setting of either polarisation controller. In no way it does mean that the value of the optically measured current depends on the fibre position within the magnetic field. This was checked by an independent set of measurements that have clearly shown a total independence of the response on the position and shape of the fibre sensing loop.

Another critical test is shown in Figure 6, in which the sensing fibre was subject to strong temperature change. Probably the same causes explain the observed changes, as in the previous situation shown in Figure 5. The increased change at low temperature may be due to an increased effective linear birefringence resulting from the reduced softness of the primary fibre coating.

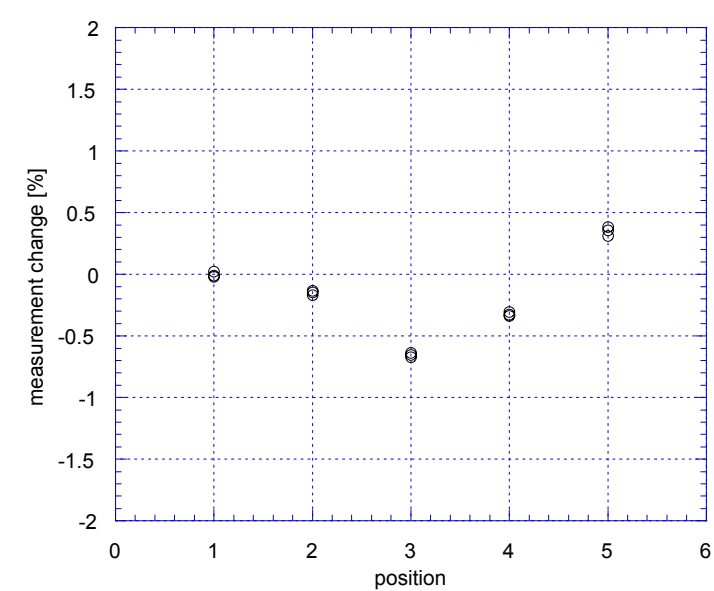

Figure 5: Measurement change for different mechanical setting of the sensing fibre.

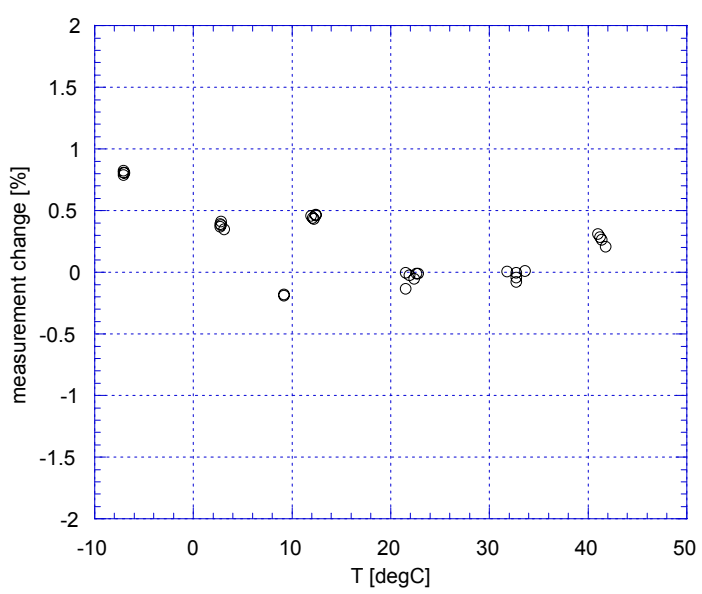

Figure 6: Measurement change as a function of the temperature of the sensing fibre cable.

Figure 7 shows the polarisation on a Poincaré sphere at the output of the sensing cable when the temperature of the entire system, i.e. including optics and electronics, is varied from 6 to $42 \operatorname{degC}$. Scale lines are separated by 10 degree in azimuth and 5 degree in elevation. It must be pointed out that the measured points are not located near the pole of the sphere as expected for a circular polarisation. Actually the polarisation is really circular into the sensing head, but the polarisation is later transformed by the connectors and, to a larger extent, by the fibre pigtail leading to the polarisation analyser. Excluding this artefact, this measurement shows that the state of polarisation after propagation throughout the sensor head is reasonably stable with respect to temperature. 


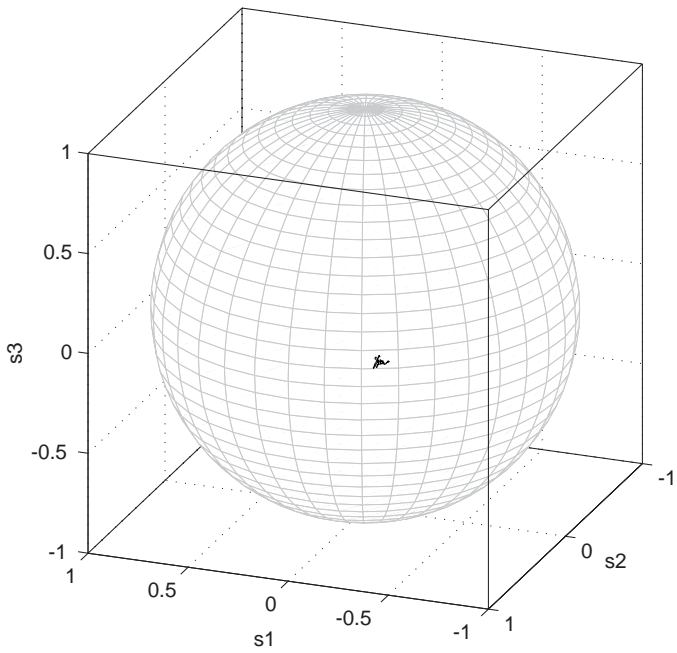

Figure 7: Output polarisation of the sensing cable, when temperature is varied from 6 to $42 \mathrm{degC}$.

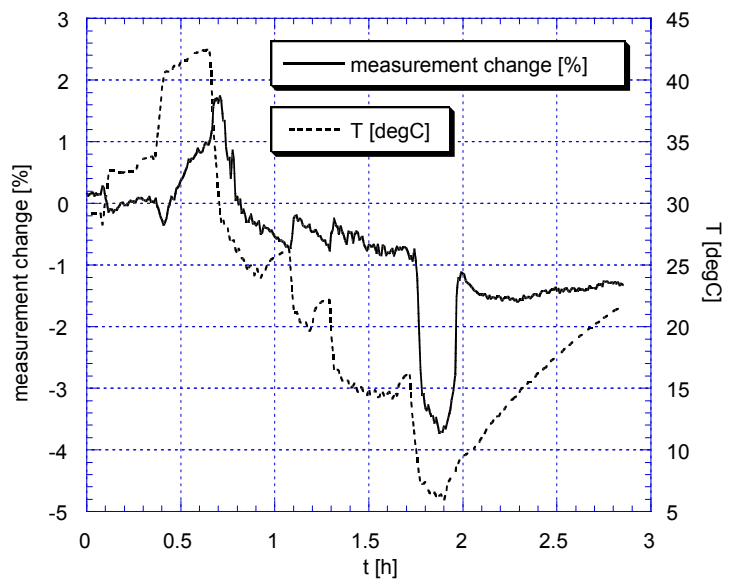

Figure 8: Measurement change as a function of time, for a thermal cycling of the complete fibre sensor.

Figure 8 shows the measurement change as a function of the case temperature, in order to evaluate the effect of temperature on the electronics and the optical elements within the sensor housing. The observed drift is mainly caused by the variation of the effective setting of the polarisation controllers, resulting in a no longer ideal polarisation within the sensing fibre. The drift of the electronics also contributes to the measurement change. The sudden shift of the measured value below $10^{\circ} \mathrm{C}$ and above $40^{\circ} \mathrm{C}$ is due to the temperature control of the Gyro electro-optic modulator that turns out to be undersized for a proper stabilisation over the entire temperature range.

\section{CONCLUSION}

This paper shows the current performances of a Sagnac interferometric current sensor in a realisation suitable for field measurements. The handling of the sensing fibre is easy and fulfils the requirement of the end user. Results are very promising and show that an accuracy of $0.5 \%$ over an extended temperature change is close to be routinely obtained. This goal would be reached by improving the electronic processing section and by further twisting the sensing fibre that still does not maintain sufficiently the circular polarisation. But most of all, these measurements demonstrate that solutions were found to solve problems that had apparently no solution for field measurements and that an accuracy of $0.5 \%$ and even $0.1 \%$ is no longer an utopia.

\section{REFERENCES}

[1] H. J. Arditty and H. C. Lefèvre, "Sagnac effect in fibre gyroscopes", Optics Letters, vol. 6, pp. 401-403, 1981

[2] W. R. Leeb, G. Schiffner and E. Scheiterer, "Optical Fibre gyroscopes: Sagnac or Fizeau effect", Applied Optics, vol. 18, pp. 1293-1295, 1979

[3] P.-A. Nicati, Ph. Robert, "Stabilized current sensor using Sagnac interferometer", Journal of Physics, vol. 21, n. 8; pp. 791-796, August 1988

[4] Z. B. Ren and Ph. Robert, "Polarization multiplexing applied to a fiber current sensor", Optics Letters, vol. 14, n. 21, pp. 1228-1230, 1989

[5] P.A. Nicati and Ph. Robert, "Stabilized Sagnac optical fibre current sensor using one phase and two amplitude modulations", $8^{\text {th }}$ OFS Proceedings, pp. 402-405, Monterey, 1992

[6] D. Tang, A. H. Rose, G. W. Day, S. M. Etzel, “Annealing of Linear Birefringence in Single-Mode Fibre Coils: Application to Optical Fibre Current Sensor", Journal of Lightwave Technology, vol. 9, n. 8, pp. 1031-1037, August 1991

[7] R. Urlich, A. Simon, "Polarization optics of twisted single-mode fibres", Applied Optics, vol. 18, n. 13, pp. 2241-2251, July 1, 1979

[8] R. Ulrich, "Fiber-optic rotation sensing with low drift", Optics Letters, vol. 5(5), pp. 173-175, 1980 